\title{
Pharmacokinetic and Pharmacodynamics Interaction between Syzygium cumini and Glipizide: Role of Cytochrome P450 Enzyme
}

\author{
Sugandha Chaudhari ${ }^{1, *}$, Shitalkumar Zambad ${ }^{2}$, Mohammed $\mathrm{Ali}^{3}$ \\ ${ }^{1}$ Department of Pharmacology, Dr. L H Hiranandani College of Pharmacy, CHM Campus, Ulhasnagar, Maharashtra, INDIA . \\ ${ }^{2}$ Thincr Technologies India Private Limited, Pune, Maharashtra, INDIA. \\ ${ }^{3}$ Phytochemistry Research Laboratory, School of Pharmaceutical Education and Research, Jamia Hamdard, New Delhi, INDIA.
}

\begin{abstract}
Background: Concomitant administration of herbs and synthetic drugs causes pharmacokinetic and pharmacodynamic interactions. Objectives: To investigate pharmacokinetic and pharmacodynamic interactions between Syzygium cumini ( $S$. cumini, SC) and Glipizide (GZ) and the role of cytochrome P450 (CYP) enzymes in interaction. Methods: The effect of the aqueous seed extract of $S$. cumini $(50$ and 100 $\mu \mathrm{g}$ ) on CYP3A was studied using in vitro liver microsomes. The interaction of GZ, a second-generation sulphonylurea, with $S$. cumini was examined in Sprague Dawley rats with type 2 diabetes induced through the administration of a high-fat diet, followed by Streptozotocin (STZ) injection. We administered $5 \mathrm{mg} / \mathrm{kg} \mathrm{GZ}$ and 250 and $400 \mathrm{mg} / \mathrm{kg} S$. cumini seeds aqueous extract orally. Pharmacodynamic parameters were evaluated by analysing blood glucose, Alanine aminotransferase (ALT) Aspartate aminotransferase (AST) and Alkaline Phosphatase (ALP) levels and by conducting the oral glucose tolerance test. The pharmacokinetics of GZ in combination with $S$. cumini was studied on the $14^{\text {th }}$ day of treatment. Plasma levels were determined through LCMS at various time points (1, 2, 4, 8 and $12 \mathrm{hr}$ after oral administration). Results: The hypoglycaemic effect exerted by the combination of $\mathrm{GZ}$ and $S$. cumini was significantly higher than that exerted by $\mathrm{GZ}$ and $S$. cumini administered alone but not below the normal level. Treatment with $G Z$ and $S$. cumini caused a significant changein ALT and ALP levels compared with treatment with GZ alone. Pre-treatment with $S$. cumini increased the AUC of GZ, which could be due to CYP3A inhibition observed in in-vitro analysis. Conclusion: Pharmacokinetic and pharmacodynamic interactions occur between S. cumini and GZ.
\end{abstract}

Key words: Herb-drug interactions, Type 2 diabetes mellitus, Pharmacokinetics, LCMS, CYP3A.

\section{INTRODUCTION}

The increasing use of herbal medicinal products in community settings in which allopathic medicine use is common may lead to serious concerns, because nearly all herbal remedies contain multiple, biologically active constituents that may interact with conventional drugs and cause adverse side effects. ${ }^{1-3}$ Many consumers believe that herbal medicines are natural and are thus safe; however, this is a dangerous over simplification. Some herbal medicines can result in undesirable effects, which include interactions with prescribed drugs. ${ }^{1-11}$ A recent survey indicated that $15 \%$ of patients who receive conventional pharmacotherapy also use herbal products and potential adverse herb-drug interactions were observed in $40 \%$ of these patients. ${ }^{12}$ In polypharmacy, determining the prevalence and rate of occurrence of drug interactions is crucial, because these interactions can have serious implications for hospitalised patients. In addition, it is important to identify and detect the major agents in herbal
Submission Date: 16-11-2018; Revision Date: 22-01-2019; Accepted Date: 30-04-2019

DOI: 10.5530/ijper.53.3s.97 Correspondence: Mrs.Sugandha Chaudhari Department of Pharmacology, Dr. L H Hiranandani College of Pharmacy, CHM Campus, Ulhasnagar- 421003, Maharashtra, INDIA. Phone: +919969033671 E-mail: chaudharisg@gmail. com

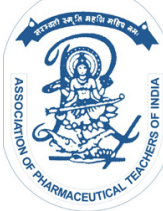

www.ijper.org 
products that cause unsafe and harmful interactions. ${ }^{13}$ Interaction of herbs with drugs is well known. Both pharmacokinetic and pharmacodynamic interactions have been reported. Pharmacodynamic interactions between drugs and herbal medicines may be attributed to an additive or synergistic effect, which can result in enhanced effects or toxicity, or to herbal medicines' antagonistic properties, which may reduce drug efficacy and cause therapeutic failure. Pharmacokinetic interactions lead to changes in the absorption, distribution, metabolism and excretion of prescribed drugs. Many drugs, such as drugs that are the substrates of Cytochrome P450 (CYP) enzymes, particularly CYP3A4 and CYP2C9, have been reported to interact with herbs. These enzymes are induced or inhibited by some routinely used herbal medicines. Accordingly, some herbal-drug interactions were reported to be mediated by CYP modulation, resulting in altered drug clearance and therapeutic effects. ${ }^{14}$ Glipizide (GZ) is a highly potent second-generation sulphonylurea derivative used to control type II diabetes mellitus. GZ is extensively metabolised in the liver by CYP2C9 and CYP3A4. ${ }^{15-19}$ Sysygium cumini (L.) (SC) Skeels (family: Myrtaceae) is a herbal drug widely used to treat diabetic conditions and is officially included in The Ayurvedic Pharmacopoeia of India. ${ }^{20}$ Jamboline, a glycoside present in the seed of SC, is considered to have antidiabetic properties. ${ }^{21}$ The seed extract of Eugenia jambolana can reduce blood glucose and glycosylated haemoglobin levels and increase the plasma insulin level. ${ }^{22}$

The present study examined the effects of the aqueous seed extract of SC on the pharmacokinetics and pharmacodynamics of GZ.

\section{MATERIALS AND METHODS}

\section{Chemicals}

We purchased glucose (Nice Chemicals Pvt. Ltd., Cochin, India); GZ (Supra Chemicals, Mumbai, India); serum AST, ALT and ALP estimation kits (Randox, Mumbai, India); and streptozotocin (STZ; HiMedia Laboratories Pvt. Ltd., Mumbai, India).

\section{In vitro assessment of CYP3A activity in liver microsomes}

After overnight fasting, rats were euthanised and their livers were perfused with $10 \mathrm{~mL}$ of $0.1 \mathrm{M}$ Phosphatebuffered Saline (PBS) and were then isolated. The liver microsomes Preparation was performed as per the method of Chaudhari et al. ${ }^{23-25}$

\section{Erythromycin-N-demethylation assay}

Estimation of CYP3A enzyme was optimised by incubating at $5 \%, 10 \% 20 \%$ and $25 \%$ of liver microsome in triplicate. Suspension is incubated with $25 \mu \mathrm{g}, 50 \mu \mathrm{g}$ and 100ug in duplicate for selection of concentration of extract. The reaction between these agents was initiated by adding NADPH $(0.1 \mathrm{~mL}, 10 \mathrm{mM})$ and terminated after $10 \mathrm{~min}$ by adding ice-cold trichloroacetic acid ( 0.5 $\mathrm{mL}, 12.5 \%, \mathrm{w} / \mathrm{v})$ solution. The mixture was then centrifuged (at $2000 \times \mathrm{g}$ for $10 \mathrm{~min}$ ) to remove proteins. Subsequently, $1.0 \mathrm{~mL}$ of Nash reagent (2M ammonium acetate, $0.05 \mathrm{M}$ glacial acetic acid and 0.02 Macetylacetone) was added to $1.0 \mathrm{~mL}$ of the supernatant; this mixture was heated in a water bath at $50^{\circ} \mathrm{C}$ for $30 \mathrm{~min}$. After cooling, absorbance was read at $412 \mathrm{~nm}$.

After stabilisation of method a mixture of microsomal suspension $(0.1 \mathrm{~mL}, 25 \%)$, erythromycin $(0.1 \mathrm{~mL}, 10$ $\mathrm{mM}$ ) and potassium phosphate $(0.6 \mathrm{~mL}, 100 \mathrm{mM}, \mathrm{pH} 7.4)$ was incubated at $37^{\circ} \mathrm{C}$ with $0.1 \mathrm{~mL}$ suspensions containing 50 and $100 \mu \mathrm{g}$ of SC; Tween $20(0.1 \mathrm{~mL}, 1 \%)$ was used as a negative control and ketoconazole $(0.1 \mathrm{~mL}$, $5 \mu \mathrm{M})$ was used as a positive control. The reaction is initiated and the absorbane was read at $412 \mathrm{~nm} .{ }^{26}$

CYP3A activity was calculated using standards (1-100 $\mathrm{mM}$ formaldehyde) prepared by substituting a sample with a standard solution, which were run in parallel. CYP3A activity is expressed as $\mathrm{nM}$ of formaldehyde $(\mathrm{CHO})$ obtained per milligram of protein per hour and was calculated using the following formula:

CYP3A activity $=$ Amount of $\mathrm{CHO}$ produced $(\mathrm{n} / \mathrm{mol})$ $\times 1 / 25 \mathrm{mg}$ of protein $\times 1 / 10 \mathrm{~min}$

\section{Experimental animals}

The experimental protocol performed on adult male Sprague-Dawley rats (weighing 180-250g) was approved by the institutional animal ethics committee and animals were maintained under standard conditions (a temperature of $22^{\circ} \mathrm{C} \pm 2^{\circ} \mathrm{C}$ and a relative humidity of $60 \% \pm$ $5 \%$ in an animal house approved by the Committee for the Purpose of Control and Supervision on Experiments on Animals (879/PO/Re/s/05/CPCSEA).

\section{Diabetes induced in rats through high-fat diet feeding and STZ administration}

Diabetes was induced in adult Sprague-Dawley albino rats through administration of a high-fat diet for 2 weeks, followed by a single intraperitoneal injection of STZ $(33 \mathrm{mg} / \mathrm{kg})$ in $0.1 \mathrm{M}$ citrate buffer $(\mathrm{pH} 4.5)$ to overnight-fasted rats (Figure 1). STZ-injected rats were allowed to drink $20 \%$ glucose solution to prevent STZinduced hypoglycaemic mortality. The threshold level 
of fasting blood glucose for diagnosing diabetes was set as $>200 \mathrm{mg} / \mathrm{dL}$. Animals received drug treatment for 14 days after diabetes induction..$^{25,27}$

\section{Treatment}

Animals were divided into six groups $(n=6$ in each group):

Group I: Vehicle (1 mL/kg, p.o.)

Group II: GZ (5 mg/kg, p.o.)

Group III: SC (250 mg/kg, p.o.) (SC 250)

Group IV: SC (400 mg/kg, p.o.) (SC 400)

Group V: SC (250 mg/kg, p.o) + GZ (5 mg/kg, p.o.) (SC $250+$ GZ)

Group VI: SC (400 mg/kg, p.o.) + GZ (5 mg/kg, p.o.) $(\mathrm{SC} 400+\mathrm{GZ})$

The seed extract of S. cumini was dissolved in $0.2 \%$ CMC for administration. To prevent any physicochemical effect on GZ absorption, GZ was administered to all animals an hour later after SC administration through gavage.

\section{Pharmacodynamic assessment}

Blood glucose, AST, ALT and ALP levels were determined on the $14^{\text {th }}$ day of drug treatment by collecting blood samples from the retro-orbital sinus under anaesthesia.

\section{Pharmacokinetic Assessment}

Plasma samples were collected after treatment with GZ at 1, 2, 4, 8 and $12 \mathrm{hr} \mathrm{10 \%} \mathrm{of} \mathrm{total} \mathrm{blood} \mathrm{volume} \mathrm{was}$ withdrawn. Plasma samples preparation and LCMS analysis of these samples was performed (SI-CART Labs, Gujarat, India) as per the method Chaudhari et al. ${ }^{25}$ The blood was mixed with a required amount of anti-coagulant $(3.8 \%$ sodium citrate) and centrifuged at $3,000 \times \mathrm{g}$ for $7 \mathrm{~min}$ to collect the plasma and stored at $-20^{\circ} \mathrm{C}$ for quantification of GZ by LC-MS. In 500ul plasma $2 \mathrm{ml}$ of ethyl acetate was added. After 2 min centrifugation, upper layer in the test tube was evaporated to dryness in nitrogen dryer. $1 \mathrm{ml}$ acetonitrile was added

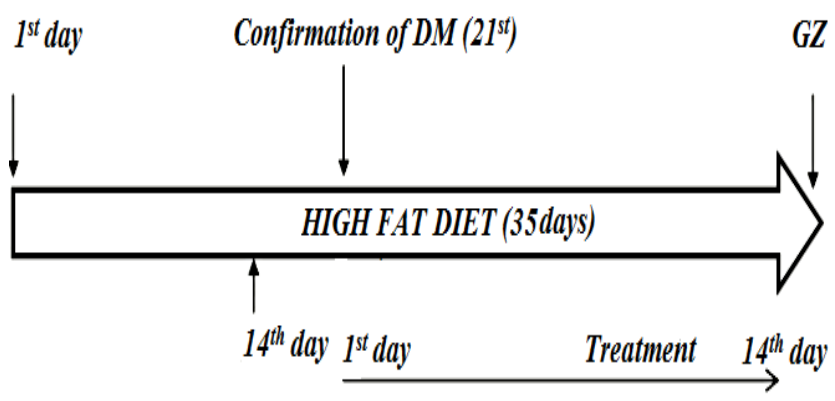

STZ (33 mg/kg, i.p.)

Figure 1: Schematic representation of the experimental. and $100 \mu \mathrm{l}$ of this solution injected in LC-MS. $10 \mathrm{mM}$ Ammonium Acetate pH-3.0: Acetonitrile (40:60) was selected as a mobile phase. A $100 \mu \mathrm{l}$ aliquot of sample was injected into a reverse phase $C_{18}$ Phenomenex column $(250 \mathrm{~mm} \times 4.6 \mathrm{~mm}$ i.d., $5 \mu \mathrm{m})$ pumped with the assigned mobile phase at a flow rate of $1 \mathrm{ml} / \mathrm{min}$. The observed peaks were well resolved and therefore the modified method was adopted for quantification of GZ in rat plasma.

\section{Oral glucose tolerance test}

The Oral Glucose Tolerance Test (OGTT) was performed in overnight-fasted rats. Glucose ( $2 \mathrm{~g} / \mathrm{kg}$ p.o.) was fed 30 min after the administration of drugs. Blood samples were collected from the retro-orbital sinus under anaesthesia after 0, 30,60 and 120 min of glucose administration and serum glucose levels were determined. ${ }^{28,29}$

\section{Statistical analysis}

Results are expressed as mean \pm S.E.M. Statistical significance was determined using one-way analysis of variance, followed by Dunnett's test.

\section{RESULTS}

\section{In-vitro assessment of CYP3A activity}

Effect of SC seed extract on liver microsomes

The in vitro effect of the SC seed extract on CYP3A activity in the liver is presented in Figure 2. We found that $50 \mu \mathrm{g}$ of the extract did not significantly reduce CYP3A activity, whereas $100 \mu \mathrm{g}$ of the extract significantly inhibited CYP3A enzyme activity $(p<0.001)$, similar to $5 \mu \mathrm{M}$ ketoconazole.

\section{Assessment of in vivo CYP3A activity}

The effect of 250 and $400 \mathrm{mg} / \mathrm{kg}$ SC on the pharmacokinetic parameters of GZ after 14 days of treatment is presented in Table 1. Compared with treatment with GZ alone, pre-treatment with 250 and $400 \mathrm{mg} / \mathrm{kg} \mathrm{SC}$ resulted in a significant increase in $\mathrm{C}_{\max }(p<0.001)$, AUC $(p<0.05)$ and $\mathrm{T}_{1 / 2}(p<0.01)$ of GZ but no significant change in Mean Residential Time (MRT).

\section{Effect of seed extract of S. cumini on the blood glucose level of STZ-induced diabetic rats}

The blood glucose level was recorded 1, 2, 4 and $8 \mathrm{hr}$ after treatment with GZ. SC oral administration at 250 and $400 \mathrm{mg} / \mathrm{kg}$ for 14 days caused a significant $(p<0.001)$ hypoglycaemic effect. SC250+GZ has SC400+GZ shown a significant $(P<0.05)$ decrease in blood glucose level at $1 \mathrm{hr}$ with SC $250+\mathrm{GZ}$ as well as at $8 \mathrm{hr}$ with SC250+GZ $(P<0.05)$ and SC400+GZ $(P<0.01)$ when 
compared to glipizide alone. In addition, administration of GZ $(5 \mathrm{mg} / \mathrm{kg})$ for 14 days caused a significant reduction $(p<0.001)$ in the blood glucose level of STZ-diabetic rats compared with diabetic untreated rats (Figure 3).

\section{Effect of seed extract of S. cumini on the OGTT}

The treatments with GZ in combination with SC resulted in a significant decrease in the serum glucose level compared with the control treatment. The com- bination of both doses of SC with GZ caused a significant decrease in serum glucose levels compared with GZ alone (Figure 4).

\section{Effect seed extract of S. cumini on ALP, AST and ALP levels}

The effects of SC alone as well as in combination with GZ on the serum AST, ALT and ALP levels of rats in all treatment groups are shown in Figure 5. SC in combination with GZ caused a dose-dependent significant $(p<0.001)$ reduction in the AST level. Compared with

\section{Table 1: Pharmacokinetic parameters of Glipizide in S. cumini pre-treated rats.}

\begin{tabular}{|c|c|c|c|}
\hline Parameter & Glipizide control & SC250+GZ & SC400+GZ \\
\hline $\mathrm{C}_{\text {max }}(\mathrm{ng} / \mathrm{ml})$ & $77.96 \pm 4.26$ & $113.6 \pm 11.65^{*}$ & $907.73 \pm 14.78^{* * *}$ \\
\hline $\mathrm{AUC}_{0-\mathrm{t}}(\mathrm{ng} / \mathrm{ml}$ h) & $570.82 \pm 10.38$ & $576.98 \pm 33.21$ & $3.46 \pm 0.07^{* *}$ \\
\hline $\mathrm{T}_{1 / 2}(\mathrm{~h})$ & $2.56 \pm 0.16$ & $2.16 \pm 0.05$ & $4 \pm 0$ \\
\hline $\mathrm{T}_{\max }(\mathrm{h})$ & $4 \pm 0$ & $4 \pm 0$ & $6.95 \pm 0.11$ \\
\hline MRT $(\mathrm{h})$ & $6.41 \pm 0.09$ & $5.6 \pm 0.11$ & \\
\hline
\end{tabular}

$C_{\max }$ : peak plasma concentration; $A U C$ : area under the plasma concentration curve; $T_{1} / 2$ : half-life; $T_{\max }:$ time to reach $C_{\max } M R T$ : mean residence time. All values are standard error mean. $a P<0.05, b P<0.01, c P<0.001$ when compared with Glipizide group (one-way ANOVA followed by Dunnett post hoc test) ( $n=3$ )

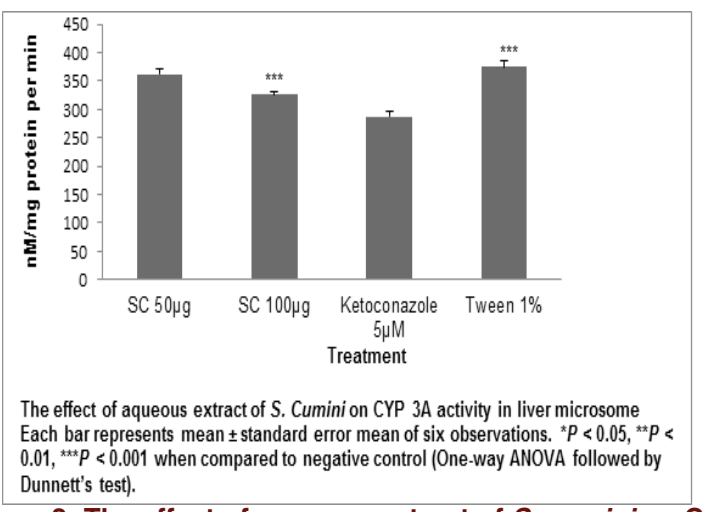

Figure 2: The effect of aqueous extract of S. cumini on CYP $3 \mathrm{~A}$ activity in liver microsome

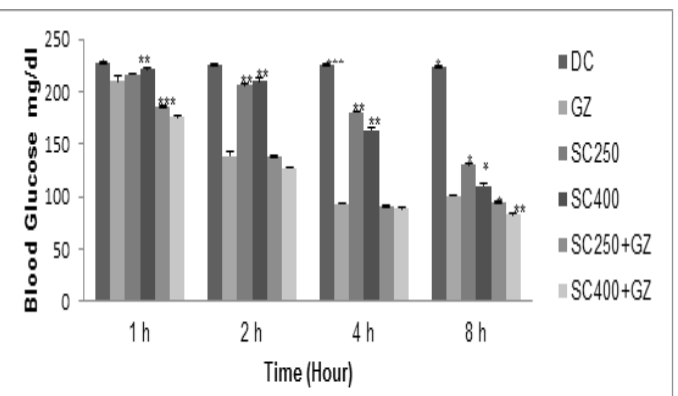

The effect of treatment of aqueous extract of $S$. Cumini on blood glucose levels in diabetic rats. All values are mean \pm S.E.M.M, $n=6 .{ }^{*} P<0.05,{ }^{*} p<0.01,{ }^{* t *} P<0.001$ when compared to GZ alone (One-way ANOVA followed by Dunnett's test).

Figure 3: The effect of treatment of aqueous extract of $S$. cumini on blood glucose levels in diabetic rats.

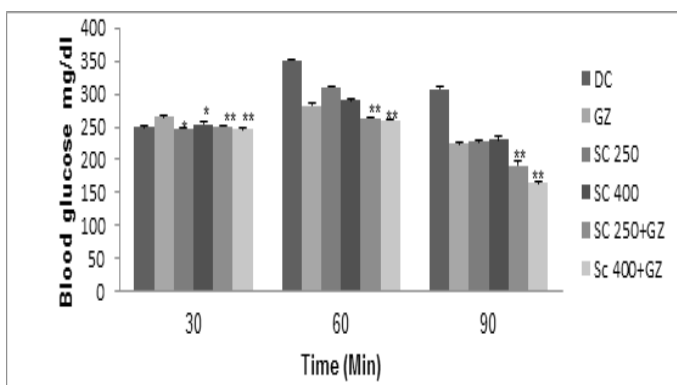

The effect of aqueous extract of $S$. cumini on oral glucose tolerance test. Each bar represents mean \pm standard error mean of six observations. ${ }^{*} p<0.05$, ${ }^{*} *<<0.01$,

${ }_{t x t} \mathrm{P}<0.001$ when compared to Glipizide alone (One-way ANOVA followed by

Figure 4: The effect of aqueous extract of $S$. cumini on oral glucose tolerance test.

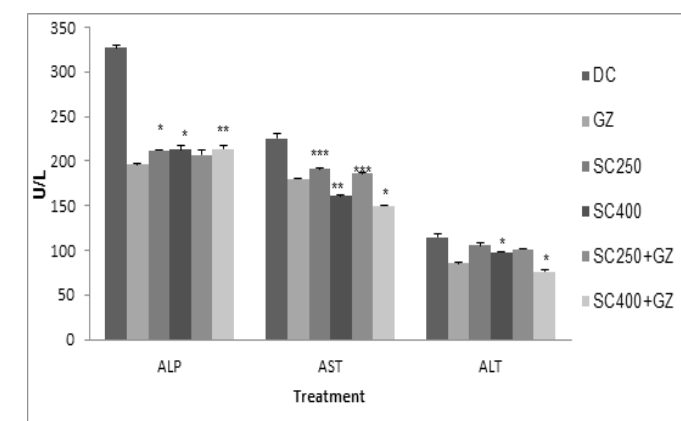

The effect of treatment of aqueous extract of S. cumini on ALP, AST, ALT levels in

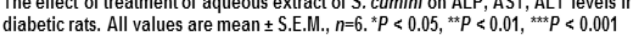
diabetic rats. All values are mean \pm S.E.M., $n=6$. ' $P<0.05$, $P<0.01$,
when compared to $G Z$ alone (One-way ANOVA followed by Dunnett's test).

Figure 5: The effect of treatment of aqueous extract of $S$. cumini on ALP, AST, ALT levels in diabetic rats. 
GZ alone, the ALT level was significantly reduced by $400 \mathrm{mg} / \mathrm{kg} \mathrm{SC}$ in combination with GZ. Furthermore, compared with GZ alone, $400 \mathrm{mg} / \mathrm{kg}$ SC in combination with $\mathrm{GZ}$ resulted in a significant $(p<0.01)$ reduction in the ALP level (Figure 5).

\section{DISCUSSION}

The use of complementary therapies for the treatment of diabetes is ever increasing and often remains unnoticed by physicians. ${ }^{30}$ Because most clinically used drugs are metabolised by CYP enzymes, changes in CYP activity initiated by herbal medications can alter the pharmacokinetics of co-administered drugs. This can subsequently reduce the pharmacological effects of co-administered drugs or cause toxicity. ${ }^{31}$ In the present study, the pharmacokinetic profile of GZ was evaluated considering that GZ is a substrate of CYP3A. We investigated the effect of SC on erythromycin-N-demethylation, which is an indicator of CYP3A activity, by using liver microsomes. The SC extract exhibited dose-dependent inhibitory effects on liver CYP3A. Ketoconazole was used as the reference standard in this study because of its reported in vitro and in vivo inhibitory effects on CYP4503A activity. ${ }^{32}$ Treatment with 250 and $400 \mathrm{mg} /$ $\mathrm{kg}$ SC for 2 weeks altered the pharmacokinetic parameters of GZ. The plasma concentration $(\mathrm{ng} / \mathrm{mL})$-time profile of GZ orally administered after SC administration increased. CYP3A inhibition through SC pre-treatment led to an increase in the $\mathrm{C}_{\max }$ and $\mathrm{AUC}$ of GZ in diabetic rats. Pre-treatment with SC also increased the half-life compared with the administration of GZ alone, confirming the occurrence of interaction between SC and GZ during metabolism. In previous studies, the aqueous seed extract of SC exerted a hypoglycaemic effect on STZ-induced diabetic rats. ${ }^{33,34}$ The seeds of SC contain glucosides, jamboline and ellagic acid, which have the ability to control the conversion of starch into sugar in case of excess production of glucose. ${ }^{35}$ In the present study, compared with GZ alone, both 250 and $400 \mathrm{mg} / \mathrm{kg}$ doses of SC in combination with GZ caused a significant decrease in the blood glucose level but no hypoglycaemia. In the OGTT test, 250 and $400 \mathrm{mg} /$ $\mathrm{kg}$ SC in combination with GZ resulted in a significant decrease in the blood glucose level. A high plasma GZ level due to pre-treatment with the SC seed extract might have shown significant alteration in glucose level in presence of combination of GZ with SC. SC seeds possess antioxidant properties. ${ }^{36}$ Treatment with 400 $\mathrm{mg} / \mathrm{kg}$ SC in combination with GZ reduced the elevated AST and ALT levels in diabetic rats compared with diabetic control rats, thereby suggesting beneficial effects of SC on altered liver function in diabetes. The results of the present study demonstrated that aqueous extract of seeds of SC interact with GZ through CYP3A inhibition by enhancing the systemic bioavailability of GZ.

\section{CONCLUSION}

The results of in vitro and in vivo studies indicated that drug interactions occurred after the co-administration of the SC extract with the CYP3A substrate agent GZ. This pharmacokinetic interaction caused a significant increase in the GZ level, resulting in pharmacodynamic interactions. Therefore, future studies should isolate different constituents of the seed extract of SC and examine their interaction with GZ.

\section{ACKNOWLEDGEMENT}

The authors are thankful to Hyderabad (Sind) National Collegiate Board and Dr Paraag Gide, Principal Dr L H Hiranandani College of Pharmacy, Ulhasnagar for providing the animal house facility for the research work.

\section{ABBREVIATIONS}

ALT: Alanine aminotransferase; ALP: Alkaline Phosphatase; AUC: Area under Curve; AST: Aspartate aminotransferase; $\mathbf{C}_{\max }$ : Maximum Measured Blood Concentration; $\mathbf{T}_{1 / 2}$ : Terminal Elimination Half-Life; $\mathbf{T}_{\max }$ : Time of Maximum Measured Plasma Concentration.

\section{REFERENCES}

1. Williamson EM. Drug interactions between herbal and prescription medicines. Drug Saf. 2003;26(15):1075-92.

2. Yang GF, Huang $X$. Development of quantitative structure-activity relationships and its application in rational drug design. Curr Pharm Des. 2006;12(35):4601-11.

3. Gardiner P, Phillips R, Shaughnessy AF. Herbal and dietary supplement: drug interactions in patients with chronic illnesses. Am Fam Physician. 2008;77(1):73-8.

4. Zhou SF, Zhou ZW, Li CG. Identification of drugs that interact with herbs in drug development. Drug Discov Today. 2007;12(15-16):664-73.

5. Lu Y, Figler B, Huang H, Tu YC, Wang J, Cheng F. Characterization of the mechanism of drug-drug interactions from PubMed using MeSH terms. Plos One. 2017;12(4):e0173548. doi:10.1371/journal.pone.0173548.

6. Tomlinson $\mathrm{B}, \mathrm{Hu} \mathrm{M}$, Lee VW. In vivo assessment of herb drug interactions: possible utility of a pharmacogenetic approach?. MolNutr Food Res. 2008;52(7):799-80.

7. DeSmet PA. Clinical risk management of herb-drug interactions. Br J Clin Pharmacol. 2007;63(3):258-67.

8. Barbara W, Leala KW, Claire G. Concurrent Use of Prescription Drugs and Herbal Medicinal Products in Older Adults: A Systematic Review. Drugs Aging. 2017;34(12):891-905.

9. Butterweck $\mathrm{V}$, Derendorf $\mathrm{H}$. Potential of pharmacokinetic profiling for detecting herbal interactions with drugs. Clin Pharmacokinet. 2008;47(6):383-97.

10. Bush TM, Rayburn KS, Holloway SW, et al. Adverse interactions between herbal and dietary substances and prescription medications: A clinical survey. Alternat Ther Health Med. 2007;13(2):30-5. 
11. Izzo AA, Ernst E. Interactions between herbal medicines and prescribed drugs: A systematic review. Drugs. 2001;61(15):2163-75.

12. Amadi CN, Mgbahurike AA. Selected Food/Herb-Drug Interactions: Mechanisms and Clinical Relevance. Am J Ther. 2018;25(4):e423-33. doi: 10.1097/MJT.0000000000000705.

13. Matheny C, Lamb M, Brouwer K, Pollack G. Pharmacokinetic and pharmacodynamic implications of P-glycoprotein modulation. Pharmacotherapy. 2001;21(7):778-96.

14. Wanwimolruk S, Phopin K, Prachayasittikul V. Cytochrome P450 enzyme mediated herbal drug interactions (Part 2). EXCLI J. 2014;13:869-96.

15. Ondieki G, Nyagblordzro M, Kikete S, Liang R, Wang L, He X. Cytochrome P450 and P-glycoprotein-mediated interactions involving African herbs indicated for common non-communicable diseases. Evidence-Based Complementary and Alternative Medicine. 2017;2017:2582463. Article ID 2582463. doi:10.1155/2017/2582463

16. Sprouse AA, Breemen RBV. Pharmacokinetic interactions between drugs and botanical dietary supplements. Drug Metab Dispos. 2016;44(2):162-71.

17. Peart GF, Boutagy J, Shenfield GM. The metabolism of glyburide in subjects of known debrisoquin phenotype. Clin Pharm Ther. 1989;20:283-95.

18. Wensing G. Glipizide: An oral hypoglycemic drug. Am J Med Sci. 1989;298(1):69-71.

19. Brian WR. Hypoglycemic agents, Metabolic drug interactions. Philadelphia: Lippincott Williams and Wilkins. 2000;429-43.

20. The Ayurvedic Pharmacopoeia of India, Government of India Ministry of Health And Family Welfare Department of Ayush. 2016;2:57. PART-1.

21. Ratsimamanga A, Loiseau S, Ratsimamanga US. Action of a hypoglycemic agent found in the young bark of Eugenia jambolania [sic] (Myrtaceae) on induced hyperglycemia of the rabbit and continuation of its purification. Comptes Rendus Hebdomadaires des Seances de l'Academie des. Sciences D: Sciences Naturelles. 1973;277(20):2219-22.

22. Ravi K, Ramachandran B, Subramanian S. Protective Effect of Eugenia jambolana seed kernel on tissue antioxidants in streptozotocin-induced diabetic rats. Biological and Pharmaceutical Bulletin. 2009;27(8):1212-7. doi:10.1248/bpb.27.1212.

23. Wrighton SA, Schuetz EG, Watkins PB, Maurel P, Barwick J, Bailey BS. Demonstration in multiple species of inducible hepatic cytochromes P-450 and their mRNAs related to the glucocorticoid-inducible cytochrome P-450 of the rat. Mol Pharmacol. 1985;28(3):312-21.

\section{PICTORIAL ABSTRACT}

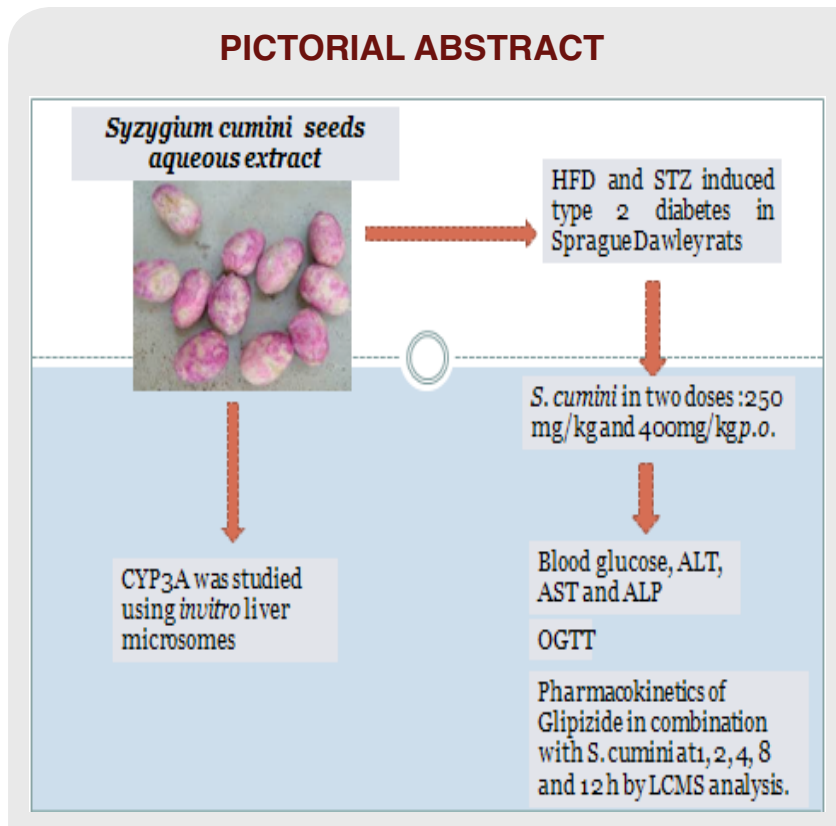

24. Umathe SN, Dixit PV, Kumar V, Bansod KU, Wanjari MM. Quercetin pretreatment increases the bioavailability of pioglitazone in rats: Involvement of CYP3A inhibition. Biochemical Pharmacology. 2008;75(8):1670-6.

25. Chaudhari S, Zambad S, Ali M. Effect of Aqueous Extract of Azadirachta indica Leaves on Pharmacokinetics and Pharmacodynamics of Glipizide. Drug Metabolism Letters. 2018. DOI : 10.2174/1872312812666181106115 247

26. Sunday ON, Mathew JO, Daniel LA, Kosisochi CA, Chinwe VU. Inhibition of cytochrome P450 3A enzyme by Millettia aboensis: its effect on the pharmacokinetic properties of efavirenz and nevirapine. Rev Bras Farmacogn. 2017;27(2):228-35.

27. Srinivasan K, Viswanad B, Asrat L, Kaul CL, Ramarao P. Combination of high-fat diet-fed and low-dose streptozotocin-treated rat: A model for type 2 diabetes and pharmacological screening. Pharmacol Res. 2005;52(4):31320.

28. Trinder P. Modified assay procedure for the estimation of serum glucose using microwell reader. Ann Clin Biochem. 1969;6:24.

29. Samala S, Veeresham C. Pharmacokinetic and pharmacodynamic interaction of boswellic acids and andrographolide with glyburide in diabetic rats: including its PK/PD modelling. Phytother Res. 2016;30(3):496-502.

30. Gurley BJ, Gardner SF, Hubbard MA, Williams DK, Gentry WB, Cui Y, et al. Cytochrome $\mathrm{P} 450$ phenotypic ratios for predicting herb-drug interactions in humans. Clin Pharmacol Ther. 2002;72(3):276-87.

31. Shi S, Klotz U. Drug interactions with herbal medicines. CliPharmacokinet. 2012;51(2):77-104.

32. Greenblatt DJ, Zhao Y, Venkatakrishnan K, Duan, SX, Harmatz JS, Parent SJ, et al. Mechanism of cytochrome P450 3A inhibition by ketoconazole. J Pharm Pharmacol. 2011;63(2):214-21.

33. Steinmetz EF. A botanical drug from the tropics used in the treatment of diabetes mellitus. Acta Phyto-therapeutica. 1960;7(2):23-5.

34. Gordon A, Jungfer E. Phenolic constituents and antioxidant capacity of four underutilized fruits from the Amazon region. Journal of Agricultural and Food Chemistry. 2011;59(14):7688-99.

35. Giri J, Sathidevi J, Dushyanth N. Effect of jamun seed extract on alloxan induced diabetes in rats. Journal of the Diabetic Association of India. 1985;25(4):115-9.

36. Prince P, Venon M. Effect of Syzigium cumini in plasma antioxidants on alloxan-induced diabetes in rats. Journal of Clinical Biochemistry and Nutrition. 1998;25(2):81-6.

\section{SUMMARY}

The use of complementary therapies for the treatment of diabetes is ever increasing and often remains unnoticed by a physician. In present study glipizide pharmacodynamic and pharmacokinetic alteration was studied in combination with Sysygium cumini. Inhibition of CYP3A activity in in-vitro analysis on liver microsome was obtained due to $S$. cumini. In high fat diet and STZ induced diabetic model, the hypoglycaemic effect observed with combination of glipizide and S. cumini was significantly high as compared to either of the drugs given alone but was not below the normal level. There was a significant decrease in ALT and ALP level compared to glipizide alone. S. cumini pre-treatment shown significant increase in AUC of glipizide, which could be due to CYP3A inhibition observed in in-vitro analysis. Data revealed that there was a pharmacokinetic and pharmacodynamic interaction between $S$. cumini with glipizide. 


\section{ABOUT AUTHORS}

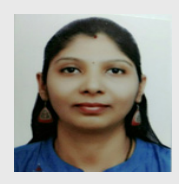

Mrs Sugandha Chaudhari, Department of Pharmacology, Dr. L H Hiranandani College of Pharmacy, Ulhasnagar, Maharashtra- 421003, India.

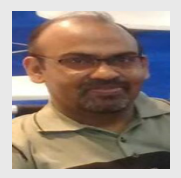

Dr Shitakumar Zambad, Thincr Technologies India Private Limited, Pune, Maharashtra, India.

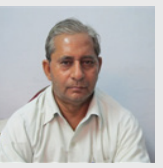

Dr Mohammed Ali, Phytochemistry Research Laboratory, School of Pharmaceutical Education and Research, Jamia Hamdard, New Delhi, India.

Cite this article: Chaudhari S, Zambad S, Ali M. Pharmacokinetic and Pharmacodynamics Interaction between Syzygium cumini and Glipizide: Role of Cytochrome P450 Enzyme. Indian J of Pharmaceutical Education and Research. 2019;53(3 Suppl 2):s273-s279. 